\title{
Eastern Woodchuck
}

National Cancer Institute

\section{Source}

National Cancer Institute. Eastern Woodchuck. NCI Thesaurus. Code C124252.

A burrowing rodent of the family Sciuridae, belonging to the group of large ground squirrels known as marmots. The woodchuck model of viral-induced hepatocellular carcinoma (HCC) provides an animal model that resembles the complex human liver environment of HCC in the context of chronic hepatitis B viral infection. 